\title{
Non-linear Thermally Induced Vibrations of Non- homogeneous Rectangular Plate of Linearly Varying Thickness in the Presence of External Force
}

\author{
Arun Kumar Gupta \\ Department of Mathematics, M.S.College, Saharanpur-247001, U.P., India.
}

\begin{abstract}
(Received 25 January 2015; accepted 21 October 2015)
An analysis with numerical results is presented for non-linear thermally induced forced vibrations of rectangular plate of variable thickness on the basis of classical plate theory. The thickness of the plate is considered as linearly varying in $\mathrm{x}$-direction. Approximate formulae are proposed for estimating the maximum deflection of a rectangular plate subject to a uniformly distributed harmonic lateral load. The effect of structural parameters such as thermal constant and taper constant with different aspect ratios on vibration of simply supported-free-simply supportedfree plate for maximum deflection for the different values of the fundamental frequency of vibration is studied. Results are presented in tabular form.
\end{abstract}

\section{INTRODUCTION}

Non-linear thermal vibration for mechanical device has been one of the hot research topics. A forced vibration device with variable thickness and non-linear thermal effect led by harmful vibration reduction is undoubtedly more economical means. The study of forced vibration behaviour of plates in the presence of non-linear thermal gradient for rectangular plates is required due to its practical importance because machines very often operate under varied temperature conditions. The temperature effects are overlooked in majority of cases though this may be a major concern for the reason that during heated up periods, structures are exposed to high intensity heat fluxes and the material properties undergo significant changes; hence, the thermal effect on modulus of elasticity of material cannot be neglected. In modern times, such materials find application because of their reduction of weight and size, low expenses, and enhancement in effectiveness and strength.

Designing and fabrication of various structures requires accuracy and perfection technically as well as economically. Though plates of variable thickness are frequently used, best fitted and suitably tapered structures are appreciated by structural engineers. It is also important for engineers to find a suitable type of tapering for their mechanical structure as variety of tapered plates are required for various types of structures and for different purposes. Therefore, the thickness variation of the plates plays a vital role in the manufacturing of the structure. Further, tapering saves weight by removing unnecessary weight of the structure. Thickness tapering is desirable since stresses tend to vary significantly within the structure.

The materials are developed depending upon the requirement and durability so that these can be used to give better strength, flexibility, weight effectiveness, and efficiency. Thus, so some new materials and alloys are utilized in making structural parts of equipment used in modern technological industries like space craft, jet engine, earth quake resistance structures, telephone industry etc. It is important that first few fre- quencies of structure be known before finalizing the design of a structure. The study of vibration of plate structures is important in a wide variety of applications in engineering design. Elastic plates are widely employed nowadays in civil, aeronautical, and marine structural designs. Complex shapes with variety of thickness variation are sometimes incorporated to reduce costly material, lighten the loads, provide ventilation, and alter the resonant frequencies of the structures.

In the recent past, a considerable amount of work has been done on vibration of plates having variable thickness, owing to their continually increasing use in the dynamic design of various engineering structures. However, no work is available on the vibration of rectangular plates of thickness variable with non-linear temperature in the presence of external force. So far only few papers have been devoted to vibration of rectangular plate of variable thickness in presence of external force. Vibration of plates of various shapes, homogeneous, orthotropic or isotropic, with or without variation in thickness, have been studied by various authors, with or without considering the effect of external force.

Akiyama and Kuroda ${ }^{1}$ discussed the fundamental frequencies of rectangular plates with linearly varying thickness. Bambill et al. ${ }^{2}$ have studied the transverse vibrations of rectangular, trapezoidal, and triangular orthotropic cantilever plates. Civalek $^{3}$ discussed the fundamental frequency of isotropic and orthotropic rectangular plates with linearly varying thickness by discrete singular convolution method. Grossi and Laura ${ }^{4}$ discussed the transverse vibrations of circular plates of linearly varying thicknesses. Gupta et al. ${ }^{5,6}$ studied the thermal effect on vibration of parallelogram plate of linearly varying thickness and bi-directional linearly varying thickness. Gupta et al. $^{7}$ studied the thermal gradient effect on vibration of a non-homogeneous orthotropic rectangular plate having bidirectional linearly thickness variation. Gupta et al. ${ }^{8}$ did the vibration analysis of visco-elastic rectangular plate with thickness varying linearly in one and parabolically in other direc- 